\title{
PIV Measurements of the Wake Formation from a Rough Flat Plate
}

\author{
S. Lawrence*, C. Atkinson, J. Soria \\ Laboratory for Turbulence Research in Aerospace and Combustion (LTRAC) \\ Department of Mechanical \& Aerospace Engineering, Monash University, Clayton, Australia ${ }^{1}$ \\ * sean.lawrence2@monash.edu
}

\begin{abstract}
Wake flows are prevalent in a wide range of engineering applications and their behaviour can significantly impact engineering design and performance. A considerable body of work exists on smooth body wake structures and flows over rough bodies, however, there is a lack of fundamental physical understanding of the amalgamation of the two fields. Two-component two-dimension particle image velocimetry (2C-2D PIV) is used to investigate the effect of surface roughness on the formation of large scale structures in the near wake of a thin flat plate. Both high-speed and low-speed, high-resolution PIV setups have been used to investigate the effect of surface roughness on the boundary layer and the near wake of the plate to gain insight into the underlying physical connection between these regions.
\end{abstract}

\section{Introduction}

Wake flows are prevalent in a wide range of engineering applications including turbomachinery, airfoil, and naval vessel design and performance. Wakes form behind any body submerged in a fluid with appreciable free steam velocity. The flow around the body and its behaviour in the wake greatly dictates the induced lift and drag. Therefore, there is significant engineering interest in understand and optimising these flows.

The presence of surface roughness introduces additional complexity to what is already a complex turbulent flow. A significant body of work exists on the impacts of surface roughness on the turbulent boundary layer (TBL), however, the effects of surface roughness on wake development have received little attention. Surface roughness on symmetric airfoils has been found to increase wake width, mean velocity deficits and turbulence intensity levels across the wake width, along with reducing the vortex shedding frequency (Zhang et al. (2004)). The underlying physical mechanism by which the modifications surface roughness makes to the TBL affect the subsequent formation of near wake structures is currently unknown.

This investigation studies the flow over smooth and two-dimensional (2D) rough surfaces using 2C-2D PIV measurements taken in the boundary layer upstream of the trailing edge and the near wake of a thin, flat plate with a blunt trailing edge.

\section{Methodology}

Experiments were performed on a series of rough acrylic plates mounted vertically on a joined aluminium spine in the LTRAC large horizontal water tunnel facility at Monash University. The roughness geometry, figure 1. consists of 2D spanwise square bars whose height increases linearly and was selected based on its elicitation of a self-preserving response in the turbulent boundary layer (Kameda et al. (2008) and Talluru et al. (2016)). For consistency, and to allow comparison with existing literature, the streamwise wavelength and relative roughness height were selected to be $\lambda_{x}=4$ and $k / \delta=0.052$, respectively. The streamwise growth rate of the roughness, $d k / d x=0.0013$, was selected using Reynolds number matching of the growth rate used by Kameda et al. (2008) with preliminary mean velocity measurements over a smooth plate. 2C2D PIV measurements were obtained using a 4 MP PCO DiMax high-speed camera and a 29 MP Imperx B6640 high-resolution camera. 


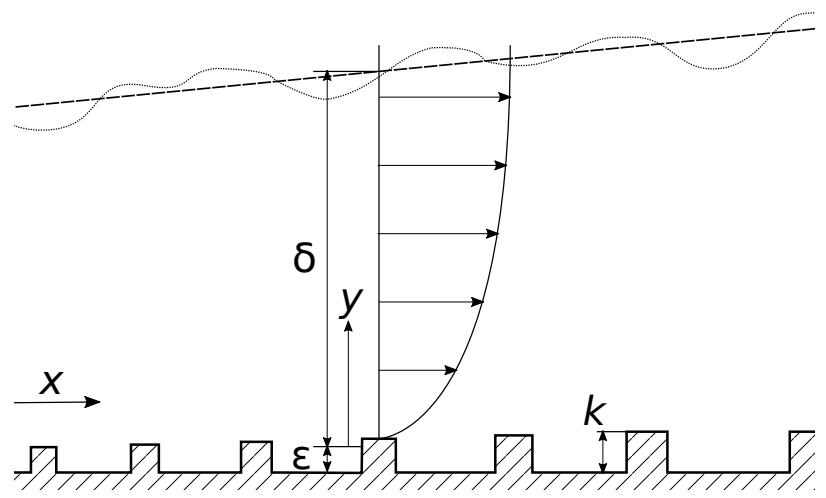

Figure 1: 2D roughness from Kameda et al. (2008).

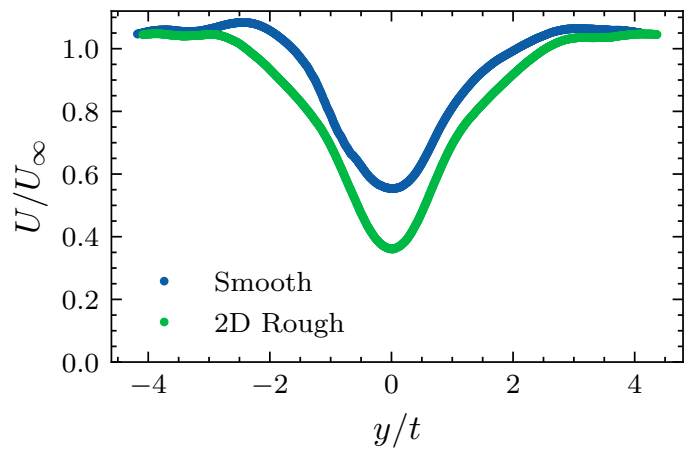

Figure 3: Near wake streamwise velocity at 4 plate thicknessess downstream $(x / t=4)$.

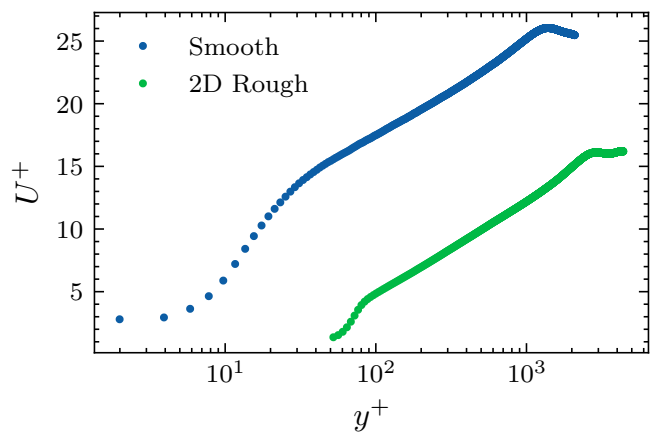

Figure 2: Mean boundary layer velocity $200 \mathrm{~mm}$ upstream of the trailing edge $\left(x_{\text {smooth }} / \delta_{99}=-3.6\right.$ and $\left.x_{\text {rough }} / \delta_{99}=-2\right)$.

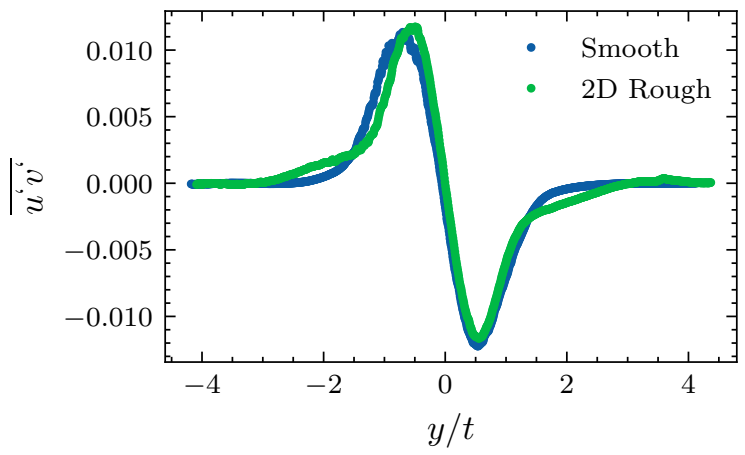

Figure 4: Near wake Reynolds stress at 4 plate thicknessess downstream $(x / t=4)$.

\section{Results}

Mean boundary layer profiles and wake profiles for the streamwise velocity and Reynolds stress are shown in figure 2, figure 3 and figure 4. These and additional results will be presented and discussed.

\section{Acknowledgements}

Financial support for this project has been provided by the Maritime Division of the Defence Science and Technology Group. The authors acknowledge the support provided by the ARC and NCMAS.

\section{References}

Kameda T, Mochizuki S, Osaka H, and Higaki K (2008) Realization of the Turbulent Boundary Layer over the Rough Wall Satisfied the Conditions of Complete Similarity and Its Mean Flow Quantities. Journal of Fluid Science and Technology 3:31-42

Soria J, Cater J, and Kostas J (1999) High resolution multigrid cross-correlation digital PIV measurements of a turbulent starting jet using half frame image shift film recording. Optics \& Laser Technology 31:3-12

Talluru KM, Djenidi L, Kamruzzaman M, and Antonia RA (2016) Self-preservation in a zero pressure gradient rough-wall turbulent boundary layer. Journal of Fluid Mechanics 788:57-69

Zhang Q, Lee SW, and Ligrani PM (2004) Effects of surface roughness and freestream turbulence on the wake turbulence structure of a symmetric airfoil. Physics of Fluids 16:2044-2053 